\title{
MERLIN 18cm observations of intermediate redshift NLS1 galaxies
}

\author{
Jens Zuther* \\ I. Physikalisches Institut, Universität zu Köln, Zülpicher Str. 77, 50937 Köln, Germany \\ E-mail: zuthereph1.uni-koeln.de

\section{Sebastian Fischer} \\ I. Physikalisches Institut, Universität zu Köln, Zülpicher Str. 77, 50937 Köln, Germany \\ E-mail: Eischerdph1.uni-koeln.de

\section{Andreas Eckart} \\ I. Physikalisches Institut, Universität zu Köln, Zülpicher Str. 77, 50937 Köln, Germany \\ E-mail: eckarteph1.uni-koeIn.de
}

We present phase-referenced MERLIN $18 \mathrm{~cm}$ observations of five $z \sim 0.2$ Narrow Line Seyfert 1 (NLS1) galaxies as part of a study of the radio properties of intermediate-redshift X-ray bright AGN. The sample is based on a match between SDSS, ROSAT, and FIRST databases. The MERLIN observations probe linear scales of about $500 \mathrm{pc}$. We do not find jet structures on kpc scales. The NLS1s turn out to have compact, radio intermediate nuclei. Using various star-formation rate indicators, we find that star formation has no significant contribution to the radio emission. This underlines the AGN nature of the compact radio emission. The radio intermediate character together with the presence of blue-/red-shifted [OIII] $\lambda 5008 \AA$ emission indicate for the presence of out-/inflows, possibly related to a radio jet in these objects. A more detailed study awaits integral field spectroscopy as well as milli-arcsecond radio observations.

Narrow-Line Seyfert 1 Galaxies and their place in the Universe - NLS1, April 04-06, 2011

Milan Italy

\footnotetext{
*Speaker.
} 


\section{Introduction}

The radio/X-ray correlation of AGN (e.g., [四]) indicates that the emission producing processes are linked, but its physical nature is still an unsolved question. The correlation is currently interpreted within the framework of the fundamental plane of supermassive black holes (e.g., [ [], [ ] ]). The model of [[] suggests a jet nature of both radio and X-ray emission, whereas the model of [B] incorporates an advection dominated accretion flow giving rise to the X-ray emission and synchrotron emission from a jet. X-ray spectral modelling and high angular resolution at radio frequencies are essential to properly disentangle accretion from jet related emission [四].

Narrow Line Seyfert 1 (NLS1) galaxies are a rare subclass of AGN. They make up to $20 \%$ in optically selected AGN samples (e.g., [[]]). The two major scenarios for explaining the NLS1 phenomenon are (i) orientation (pole on view) or (ii) young AGN growing at high rate (cf., review by [G]). As a class, NLS1s are predominantly radio quiet (RQ). Recently, however, a fraction of (very) radio loud (RL) NLS1s has been found, which makes up about 7\% of the NLS1 population [四]. RL NLS1s show close resemblance to compact steep spectrum radio sources, which are interpreted as young AGN since the last triggering. The radio loudest specimen exhibit blazar like radio properties [四]. NLS1s have quite complex X-ray spectra. In general, they are steeper than those of broad-line AGN [6]. The details of processes driving the radio and X-ray properties of NLS1 are still being explored.

In this context, we studied a small sample of 13 intermediate redshift $(0.11 \lesssim z \lesssim 0.37)$ AGN with MERLIN at $18 \mathrm{~cm}$. The linear scales probed with MERLIN at $18 \mathrm{~cm}$ are about $500 \mathrm{pc}$ (at the average redshift of $z \sim 0.2$ ). The sample is based on a crossmatch between SDSS, ROSAT, and FIRST public databases. A detailed description and discussion will be presented in [8]. In the following, we will focus on the five NLS1 galaxies in our sample. They have black hole masses in the range $\log \left(M_{\bullet} / M_{\odot}\right)=6.5-8.0$ and radio loudnesses in the range $\log R^{*}=0.6-0.8$.

\section{Results}

We find that the MERLIN detected $18 \mathrm{~cm}$ emission is compact, with no indication for extended collimated jet structures. As compared to the VLA data, on average $35 \%$ of the FIRST emission is resolved out by MERLIN. In our data, we can, however, not discern whether the extended emission is related to a diffuse star-formation or jet component. For one source, we find strongly increased emission, which clearly indicates for variability. Also, we cannot dismiss the possibility of variability in the apparently resolved sources. The average brightness temperature of the compact emission in our MERLIN maps is $T_{b} \approx 2.2 \times 10^{4} \mathrm{~K}$. Such could still be consistent with a stellar origin. We used various star-formation rate indicators from the radio through optical to the X-ray (e.g., [Q]]) and found that the star formation in the hosts does not play an important role in producing the radio emission. There might be one exception, in which about $70 \%$ of the FIRST flux is resolved out by MERLIN (ignoring variability at this point). However, the induced star-formation rates are not consistent across the used wavelengths. All this indicates that the compact radio emission in these NLS1s is closely connected to processes that occur in the vicinity of the central supermassive black hole. 




Figure 1: Black-hole mass weighted $5 \mathrm{GHz}$ luminosity versus the soft X-ray luminosity in units of the Eddington luminosity. See text for details.

The distribution of various samples of galactic and extragalactic sources within the 3D space of the fundemantal plane $\left(L_{X}, L_{R}, M_{\bullet}\right)$ is used to study the underlying physical processes that give rise to the apparent correlation between X-ray and radio emission. In Fig. W, we present a projection of the fundamental plane according to [B] (their Fig. 7). The data points are color coded with their radio loudness, $R_{X}$, as measured by the ratio of radio over X-ray flux density [प]]. [प] defines the demarcation between RL and RQ objects empirically to be around $\log R_{X}=-2.5$. Our data are given as dark grey circles with borders colored according to their $R_{X}$. For comparison, we include further data sets from the literature: The data of [B] are given as asterixs, SDSS/ROSAT/FIRST broad-line AGN of [ए2] are shown as '+' symbols, low luminosity AGN by [एँ3] as downright triangles, low luminosity Seyfert 1,2 galaxies from [ए]] as hexagons, soft X-ray selected NLS1s from [14] as diamonds, radio galaxies from [15]] as crosses, and mas angular resolution studies by [ㅈ] as upright triangles and [ㄱ] as pentagons. The dashed black line is the fit of [B]. Dotted and dashed-dotted lines are representing constant $\log R_{X}$ (red: -1.5 , blue: -4.5 ) for $10^{6} M_{\odot}$ and $10^{9} M_{\odot}$ black holes, respectively.

The scatter in Fig. $\square$ is considerable. There are indications that the distributions are somewhat steeper than the Merloni et al. fit. This might point towards a jet nature of radio and X-ray emission (cf., [[1]]). If, however, samples are selected within a narrow range of radio loudness, this automatically results in a slope (close to unity) steeper than the Merloni et al. fit. This has to be considered, when interpreting the data distibution. As pointed out by [四], it is furthermore 
important to better understand the complex X-ray spectra by modelling accretion and jet related emission. Such a decomposition has been shown to move the data points considerably along the horizontal axis.

Our data are following the trends seen for the BLAGN sample of [ए2] and the soft X-ray selected NLS1s of [14]. They are accreting very efficiently, their Eddington ratio, $\eta$, being about $\eta=L_{\text {bolometric }} / L_{\text {Eddington }} \approx 0.5$. The steepening towards high accretion rates $\left(\log L_{X} / L_{\text {Edd }} \rightarrow 0\right)$ might either indicate for an additional jet contribution at radio frequencies to the otherwise most efficiently accreting black hole, or for the jet origin of both X-ray and radio emission. A definite answer awaits higher angular resolution radio mapping (preferably at several frequencies). For instance, in their milli-arcsecond VLBI observations of RL NLS1s, [ए7] find strong indications for jet-induced Doppler boosting, due to very high brightness temperatures. More detailed X-ray spectroscopy would then be required to test for a possible jet contribution to the X-ray emission (cf., [[ष]]). Combined with intergal field spectroscopy in the optical (since most of the NLS1 in this sample show blue-/red-shifted [OIII] emission indicating for a complex narrow-line region kinematics), this could shed light on the presence and importance of outflows, possibly related to jets in NLS1s.

\section{Acknowledgments}

This work has benefited from research funding from the European Community's sixth Framework Programme under RadioNet R113CT 2003 5058187. MERLIN is a National Facility operated by the University of Manchester at Jodrell Bank Observatory on behalf of STFC. This work hase been supported in part by DFG SFB 494 and SFB 956. JZ acknowledges support from the European project EuroVO DCA under the Research e-Infrastructures area.

\section{References}

[1] Brinkmann, W., Laurent-Muehleisen, S. A., Voges, W., Siebert, J., Becker, R. H., Brotherton, M. S., White, R. L., \& Gregg, M. D. : Radio and X-ray bright AGN: the ROSAT - FIRST correlation, A\&A 356 (2000) 445

[2] Falcke, H., Körding, E., \& Markoff, S.: A scheme to unify low-power accreting black holes. Jet-dominated accretion flows and the radio/X-ray correlation, A\&A 414 (2004) 895

[3] Merloni, A., Heinz, S., \& di Matteo, T.: A Fundamental Plane of black hole activity, MNRAS 345 (2003) 1057

[4] Hardcastle, M. J., Evans, D. A., \& Croston, J. H.: The active nuclei of $z<1.03$ CRR radio sources, MNRAS 396 (2009) 1929

[5] Zhou, H., Wang, T., Yuan, W., Lu, H., Dong, X., Wang, J., \& Lu, Y.: A Comprehensive Study of 2000 Narrow Line Seyfert 1 Galaxies from the Sloan Digital Sky Survey. I. The Sample, ApJS 166 (2006) 128

[6] Komossa, S.: Narrow-line Seyfert 1 Galaxies, Revista Mexicana de Astronomia y Astrofisica Conference Series 32 (2008) 86

[7] Yuan, W., Zhou, H. Y., Komossa, S., Dong, X. B., Wang, T. G., Lu, H. L., \& Bai, J. M.: A Population of Radio-Loud Narrow-Line Seyfert 1 Galaxies with Blazar-Like Properties?, ApJ 685 (2008) 801 
[8] Zuther, J., Fischer, S., Eckart, A. , submitted to A\&A (2011)

[9] Kennicutt, R. C., Jr.: Star Formation in Galaxies Along the Hubble Sequence, ARA\&A 36 (1998) 189

[10] Terashima, Y., \& Wilson, A. S.: Chandra Snapshot Observations of Low-Luminosity Active Galactic Nuclei with a Compact Radio Source, ApJ 583 (2003) 145

[11] Panessa, F., Barcons, X., Bassani, L., Cappi, M., Carrera, F. J., Ho, L. C., \& Pellegrini, S.: The X-ray and radio connection in low-luminosity active nuclei, A\&A 467 (2007) 519

[12] Li, Z.-Y., Wu, X.-B., \& Wang, R.: The Black Hole Fundamental Plane: Revisited with a Larger Sample of Radio and X-Ray-emitting Broad-Line AGNs, ApJ 688 (2008) 826

[13] Yuan, F., Yu, Z., \& Ho, L. C.: Revisiting the "Fundamental Plane" of Black Hole Activity at Extremely Low Luminosities, ApJ 703 (2009) 1034

[14] Grupe, D., Wills, B. J., Leighly, K. M., \& Meusinger, H.: A Complete Sample of Soft X-Ray-Selected AGNs. I. The Data, AJ 127 (2004) 156

[15] Balmaverde, B., \& Capetti, A.: The host galaxy/AGN connection in nearby early-type galaxies. Is there a miniature radio-galaxy in every "core" galaxy?, A\&A 447 (2006) 97

[16] Ulvestad, J. S., Antonucci, R. R. J., \& Barvainis, R.: VLBA Imaging of Central Engines in Radio-Quiet Quasars, ApJ 621 (2005) 123

[17] Doi, A., et al.: Japanese VLBI Network Observations of Radio-Loud Narrow-Line Seyfert 1 Galaxies, PASJ 59 (2007) 703

[18] Körding, E., Falcke, H., \& Corbel, S.: Refining the fundamental plane of accreting black holes, A\&A 456 (2006) 439 